\title{
MIMO Indoor Propagation: A Geometry-Based Model Including Time-Variant Fading Statistics
}

\author{
Evgenii Vinogradov ${ }^{1}$, Wout Joseph ${ }^{2}$, Claude Oestges ${ }^{1}$ \\ ${ }^{1}$ ICTEAM, Université catholique de Louvain, Belgium, Evgenii.Vinogradov@UCLouvain.be \\ ${ }^{2}$ INTEC, Ghent University/iMinds, Belgium, Wout.Joseph@INTEC.UGent.be
}

\begin{abstract}
A geometry-based reference model for Second Order Scattering Fading (SOSF) distributed multiple-input-multipleoutput (MIMO) channels is proposed for mobile-to-mobile scenarios. From this model, a spatial correlation function is derived. The time-variant nature of channel statistics is modeled by using a Hidden Markov Model (HMM) based approach. The proposed method enables to implement a generator of channel realizations for a wide range of indoor MIMO channels, including Fixedto-Mobile and Mobile-to-Mobile transmission; Rician, Rayleigh, Double Rayleigh, Second Order Scattering fading, etc.
\end{abstract}

Index Terms-Indoor propagation, MIMO.

\section{INTRODUCTION}

Predicting the parameters of indoor MIMO channels is important for the testing and the eventual rollout of new communication systems. New personal communication services are indeed devoted to indoor environments, as more people spend time in offices, classrooms, stores, etc. The growing interest in wireless indoor communication systems has resulted in many papers on the characteristics of indoor radio propagation channels. In [1], peer-to-peer channels were investigated in a typical US office environment, consisting in a large indoor area containing individual cubicle-style offices. An empirical model of time-variant channel statistics of a single-input-single-output (SISO) narowband indoor channel was presented in [2].

Various geometry-based models of correlated Rayleigh MIMO channels were proposed in [3], [4]. However, as it is shown in [2], the channel has a rather mixed distribution, including the weighted combination of a line-of-sight (LOS) component, a Rayleigh fading component and a DoubleRayleigh fading component. This mixed type of fading can be described by the so-called Second Order Scattering Fading (SOSF) distribution [1], [5].

In this paper, we propose a geometry-based reference model for narroband indoor SOSF MIMO channels with time-variant first- and second-order statistics. Second-order statistics are derived from the proposed reference model, while the channel non-stationarity is modeled using a Hidden Markov Model (HMM).

\section{Model For MiMO Mobile-To-Mobile Channels}

\section{A. SOSF distributed channels}

It is shown in [1], [2] that fading can be described by a single distribution including a weighted combination of a line- of-sight (LOS) component, a Rayleigh fading component and a Double-Rayleigh fading component. Hence, any realization of the channel can be expressed as

$$
h(t)=\omega_{0} e^{j \theta}+\omega_{1} h_{1}(t)+\omega_{2} h_{2}(t) h_{3}(t)
$$

where $h_{1}, h_{2}, h_{3}$ are i.i.d. complex normal random variables with zero mean and unit variance, and $\theta$ is a constant phase shift angle in $[0,2 \pi]$. Weights $\omega_{0}, \omega_{1}$ and $\omega_{2}$ describe the impact of the LOS term, Rayleigh and Double Rayleigh components, respectively. The probability density function of $g=|h|$ is given, as shown in [5] and [1], by the SOSF distribution:

$$
\rho_{S O S F}(g)=\int_{0}^{\infty} \omega e^{-\omega_{1}^{2} \omega^{2} / 4} \frac{4 J_{0}(g \omega) J_{0}\left(\omega_{0} \omega\right)}{4+\omega_{2}^{2} \omega^{2}} d \omega
$$

where $J_{0}$ is the Bessel function of the first kind and zeroth order.

Since $\mathbb{E}\left\{g^{2}\right\}=1$, we have that $\omega_{0}^{2}+\omega_{1}^{2}+\omega_{2}^{2}=1$ and we can specify the distribution by two parameters [6]

$$
\begin{aligned}
& \alpha=\frac{\omega_{2}^{2}}{\omega_{0}^{2}+\omega_{1}^{2}+\omega_{2}^{2}} \\
& \beta=\frac{\omega_{0}^{2}}{\omega_{0}^{2}+\omega_{1}^{2}+\omega_{2}^{2}}
\end{aligned}
$$

where $(\alpha, \beta)$ are constrained to the triangle $\alpha \geq 0, \beta \geq 0$, and $\alpha+\beta \leq 1$

\section{B. Generalized Reference Double-Ring Model for SOSF chan- nels}

In this paper we consider a narrowband MIMO communication system with $L_{T}$ transmit and $L_{R}$ receive omnidirectional antenna elements.

It is shown in [3] that the double-ring geometry can be used to model MIMO channels in indoor environments. The geometry of the proposed model is shown in Fig. $1 a$ and $b$ for the cases of double- and single-bouncing, respectively. Transmitter $(\mathrm{Tx})$ and receiver $(\mathrm{Rx})$ are denoted by $0_{T}$ and $0_{R}$, respectively; $R_{T}$ and $R_{R}$ are radii of rings which are formed by scatterers $S_{T}^{m}(m=1,2, \ldots M)$ and $S_{R}^{n}(n=1,2, \ldots N)$ located around the transmitter and the receiver. The spacing between two adjacent antenna elements at the Tx and $\mathrm{Rx}$ is denoted by $\delta_{T}$ and $\delta_{R}$. Angles $\alpha_{T}^{m}$ and $\alpha_{T}^{n}$ denote the angles of departure (AoD) of the transmitting waves scattered from the scatterers $S_{T}^{m}$ and $S_{R}^{n}$, whereas $\alpha_{R}^{m}$ and $\alpha_{R}^{n}$ are the angles of arrival (AoA) of the receiving waves that impinge on $S_{T}^{m}$ and 


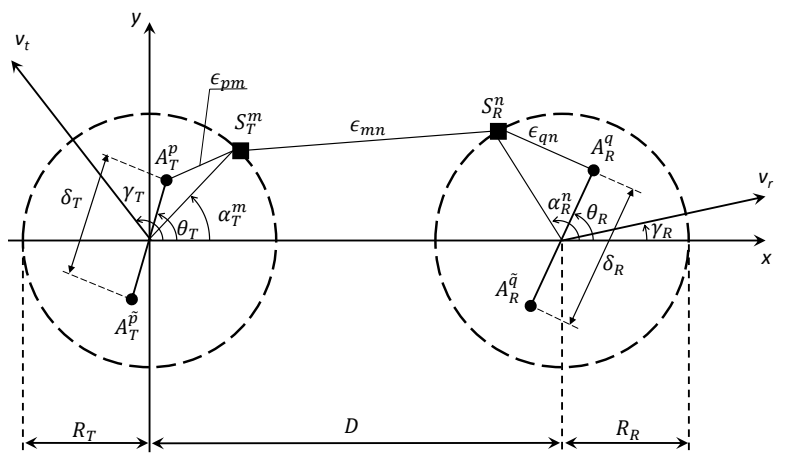

(a) Double Bouncing

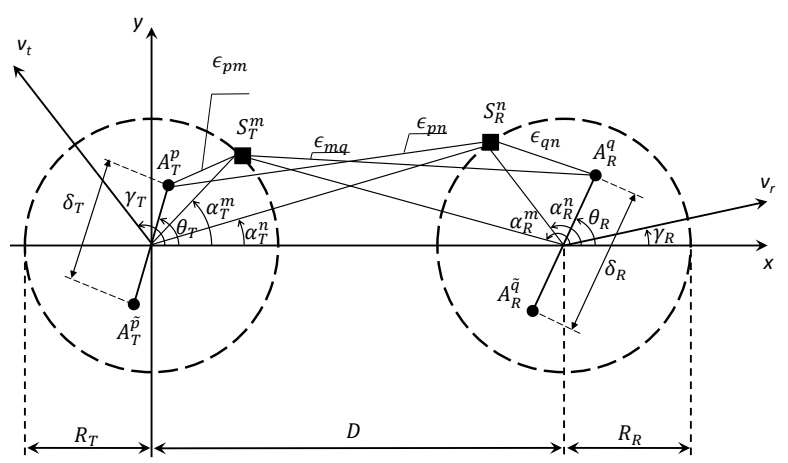

(b) Single Bouncing

Fig. 1. The geometrical double-ring model

$S_{R}^{n}$, respectively. Angles $\theta_{T}$ and $\theta_{R}$ describe the orientation of the Tx and Rx antenna array, respectively, relative to the $\mathrm{x}$-axis.

The following assumptions are made: (i) the $\mathrm{Tx}$ and $\mathrm{Rx}$ are moving with speeds $v_{t}$ and $v_{r}$ in directions described by angles $\gamma_{T}$ and $\gamma_{R}$ for the transmitter and the receiver, respectively; (ii) the distance between $\mathrm{Tx}$ and $\mathrm{Rx}$ is much larger than the radii $R_{T}$ and $R_{R}$, i.e. $\max \left(R_{T}, R_{R}\right) \ll D$; (iii) $\delta_{T}$ and $\delta_{R}$ are much smaller than the radii $R_{T}$ and $R_{R}$, i.e., $\max \left\{\delta_{T}, \delta_{R}\right\} \ll \min \left\{R_{T}, R_{R}\right\}$; (iv) the angles $\alpha_{T}^{m}$ and $\alpha_{R}^{n}$ are uniformly distributed random variables; (v) for short periods of time, the mobile environment can be assumed as quasi-stationary, i.e. the rings of scatterers are fixed; (vi) waves reaching the receiver antenna array are equal $\frac{1}{\sqrt{N(M)}}$ or $\frac{1}{\sqrt{N M}}$ for single- and double-bounce scattering, respectively.

The expressions for the distances which a plane wave travels in the case of so-called double bounce scattering $\left(\epsilon_{p m}, \epsilon_{m n}, \epsilon_{q n}\right.$ in Fig. $\left.1 a\right)$ and single bounce scattering $\left(\epsilon_{p m}, \epsilon_{m q}, \epsilon_{p n}, \epsilon_{q n}\right.$ in Fig. $\left.1 b\right)$ can be found in [3].

The complex faded envelop of the link from the transmit antenna element $A_{p}$ to the receive antenna element $A_{q}$ can be expressed as:

$$
\begin{aligned}
h^{p q}(t) & =\omega_{0} h_{L O S}^{p q}(t)+\omega_{S B R} h_{S B R}^{p q}(t)+\omega_{S B T} h_{S B T}^{p q}(t) \\
& +\omega_{D B} h_{D B}^{p q}(t)+\omega_{D B I} h_{D B I}^{p q}(t),
\end{aligned}
$$

where $h_{L O S}^{p q}(t)$ denotes the LOS term; $h_{S B R}^{p q}(t)$ and $h_{S B T}^{p q}(t)$ are the components caused by single bounce scattering at the scatterers around the receiver and the transmitter, respectively; $h_{D B}^{p q}(t)$ and $h_{D B I}^{p q}(t)$ are the components caused by double bounce scattering under conditions of correlated and independent ${ }^{1}$ scattering, respectively. The various $\omega$ are the corresponding weights.

By proceeding as in [3], we can express the components of (5) as:

$$
\begin{gathered}
h_{L O S}^{p q}(t)=e^{j 2 \pi t f_{\text {Tmax }} \cos \left(\pi-\alpha_{R q}^{L O S}-\gamma_{T}\right)} \\
\times e^{j 2 \pi t f_{R \max } \cos \left(\alpha_{R q}^{L O S}-\gamma_{R}\right)} \\
h_{S B T}^{p q}(t)=\lim _{M \rightarrow \infty} \frac{1}{\sqrt{M}} \sum_{m=1}^{M} e^{j \phi_{m}-j \frac{2 \pi}{\lambda}\left(\epsilon_{p m}+\epsilon_{m q}\right)} \\
\times e^{j 2 \pi t\left(f_{T \max } \cos \left(\alpha_{T}^{m}-\gamma_{T}\right)+f_{R \max } \cos \left(\alpha_{R}^{m}-\gamma_{R}\right)\right)} \\
h_{S B R}^{p q}(t)=\lim _{N \rightarrow \infty} \frac{1}{\sqrt{N}} \sum_{n=1}^{N} e^{j \phi_{n}-j \frac{2 \pi}{\lambda}\left(\epsilon_{p n}+\epsilon_{n q}\right)} \\
\times e^{j 2 \pi t\left(f_{T \max } \cos \left(\alpha_{T}^{n}-\gamma_{T}\right)+f_{R \max } \cos \left(\alpha_{R}^{n}-\gamma_{R}\right)\right)} \\
h_{D B}^{p q}(t)=\lim _{\substack{N \rightarrow \infty \\
M \rightarrow \infty}} \frac{1}{\sqrt{M N}} \sum_{n=1}^{N} \sum_{M=1}^{M} e^{j \phi_{m n}-j \frac{2 \pi}{\lambda}\left(\epsilon_{p m}+\epsilon_{m n}+\epsilon_{n q}\right)} \\
\times e^{j 2 \pi t\left(f_{T \max } \cos \left(\alpha_{T}^{m}-\gamma_{T}\right)+f_{R \max } \cos \left(\alpha_{R}^{n}-\gamma_{R}\right)\right)}
\end{gathered}
$$

By using the results in [2], [7] for the case of independent scatterers, we obtain

$$
\begin{aligned}
& h_{D B I}^{p q}(t)= \\
& \lim _{N \rightarrow \infty} \frac{1}{\sqrt{N}} \sum_{n=1}^{N} e^{j\left(\phi_{n}-\frac{2 \pi}{\lambda} \epsilon_{n q}\right)} \times e^{j 2 \pi t f_{R \max } \cos \left(\alpha_{R}^{n}-\gamma_{R}\right)} \\
& \times \lim _{M \rightarrow \infty} \frac{1}{\sqrt{M}} \sum_{m=1}^{M} e^{j\left(\phi_{m}-\frac{2 \pi}{\lambda} \epsilon_{p m}\right)} \times e^{j 2 \pi t f_{\text {Tmax }} \cos \left(\alpha_{T}^{m}-\gamma_{T}\right)}
\end{aligned}
$$

Since the central limit theorem states that each single sum is a zero-mean complex Gaussian process with unit variance, the envelopes $g_{D B}^{p q}(t)=\left|h_{D B}^{p q}(t)\right|, g_{S B R}^{p q}(t)=\left|h_{S B R}^{p q}(t)\right|$ and $g_{S B T}^{p q}(t)=\left|h_{S B T}^{p q}(t)\right|$ are all characterized by Rayleigh distributions whereas the envelope $g_{D B I}^{p q}(t)=\left|h_{D B I}^{p q}(t)\right|$ enables to reproduce the Double-Rayleigh distribution, so that $\omega_{D B I}$ can be identified to $\omega_{2}$ in (1). The Rayleigh term in (1) is made of the combination of all other components. Proceeding as in [2], we may set $\omega_{D B}=\omega_{S B T}=\omega_{S B R}=\frac{\omega_{1}}{\sqrt{3}}$, assuming that all three mechanisms are occurring with the same probability. Finally, $\omega_{L O S}=\omega_{0}$, so that we can express (5) as

$$
\begin{aligned}
h^{p q}(t) & =\omega_{0} h_{L O S}^{p q}(t) \\
& +\frac{\omega_{1}}{\sqrt{3}} \cdot\left(h_{D B}^{p q}(t)+h_{S B T}^{p q}(t)+h_{S B R}^{p q}(t)\right) \\
& +\omega_{2} \cdot h_{D B I}^{p q}(t) .
\end{aligned}
$$

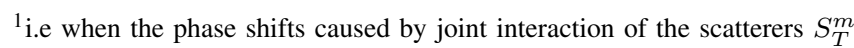
and $S_{R}^{n}$ can be expressed as $\phi_{m n}=\phi_{m}+\phi_{n}$ 
Using the model in [2], time-variant statistics of the SOSF channel (i.e weights $\omega_{0}, \omega_{1}$ and $\omega_{2}$ ) can be modeled.

\section{Statistics of AoA and AoD}

Using the geometrical model in Fig. 1, the AoD and AoA probability density functions can be derived. The PDFs of AoA and AoD in the case of double bouncing are trivial and can be expressed as

$$
f\left(\alpha_{R}^{n}\right)=f\left(\alpha_{T}^{m}\right)=\frac{1}{2 \pi}
$$

In the case of single bouncing at scatterers around the receiver $\left(h_{S B R}^{p q}(t)\right)$, the AoA PDF is described by (12), whereas the AoD PDF is described by

$$
f\left(\alpha_{T}^{n}\right)= \begin{cases}\frac{1}{\arcsin \left(\frac{R_{R}}{D}\right)} & -\alpha_{A o D}<\alpha_{T}^{n}<\alpha_{A o D} \\ 0 & \text { otherwise }\end{cases}
$$

where $\alpha_{A o D}=\arcsin \left(\frac{R_{R}}{D}\right)$.

In the case of single bouncing at scatterers around the transmitter $\left(h_{S B T}^{p q}(t)\right)$, the AoD PDF is described by (12); the AoA PDF expressed as

$$
f\left(\alpha_{R}^{m}\right)= \begin{cases}\frac{1}{\arcsin \left(\frac{R_{T}}{D}\right)} & -\alpha_{A o A}<\pi-\alpha_{R}^{m}<\alpha_{A \circ A} \\ 0 & \text { otherwise }\end{cases}
$$

where $\alpha_{A o A}=\arcsin \left(\frac{R_{T}}{D}\right)$.

As it is assumed that the total power of all waves reaching the receiver antenna array depends on the weights $\omega_{D B}, \omega_{S B T}, \omega_{S B R}, \omega_{D B I}$, we can easily obtain Power Angular Spectra using appropriate weights together with the PDFs of AoA and AoD.

\section{Spatial Correlation Function}

Using the space-time correlation function derived in [8] with appropriate parameters, the normalized spatial correlation function between two complex fading envelopes $h^{p q}(t)$ and $h^{\tilde{p} \tilde{q}}(t)$ can be written as

$$
\begin{aligned}
& R^{p q, \tilde{p} \tilde{q}}\left(\delta_{T}, \delta_{R}\right)=\omega_{0}^{2} \cdot R_{L O S}^{p q, \tilde{p} \tilde{q}}\left(\delta_{T}, \delta_{R}\right) \\
& +\frac{\omega_{1}^{2}}{3} \cdot\left[J_{0}\left(2 \pi(\tilde{p}-p) \frac{\delta_{T}}{\lambda}\right)+J_{0}\left(2 \pi(\tilde{q}-q) \frac{\delta_{R}}{\lambda}\right)\right. \\
& \left.\quad+J_{0}\left(2 \pi(\tilde{p}-p) \frac{\delta_{T}}{\lambda}\right) J_{0}\left(2 \pi(\tilde{q}-q) \frac{\delta_{R}}{\lambda}\right)\right] \\
& \quad+\omega_{2}^{2} \cdot J_{0}\left(2 \pi(\tilde{p}-p) \frac{\delta_{T}}{\lambda}\right) J_{0}\left(2 \pi(\tilde{q}-q) \frac{\delta_{R}}{\lambda}\right),
\end{aligned}
$$

where

$$
\begin{gathered}
R_{L O S}^{p q, \tilde{p} \tilde{q}}\left(\delta_{T}, \delta_{R}\right)=e^{\frac{j 2 \pi}{\lambda}\left(\delta_{T} \cos \theta_{T}-\delta_{R} \cos \theta_{R}\right)} \\
\times e^{j 2 \pi\left(f_{\text {Tmax }} \cos \gamma_{T}-f_{R \max } \cos \gamma_{R}\right)}
\end{gathered}
$$

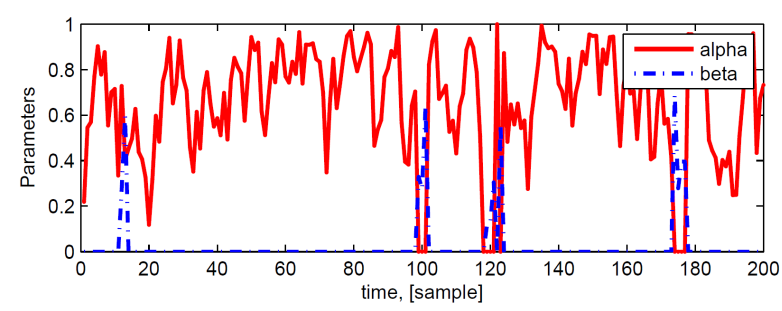

(a) SOSF distribution parameters

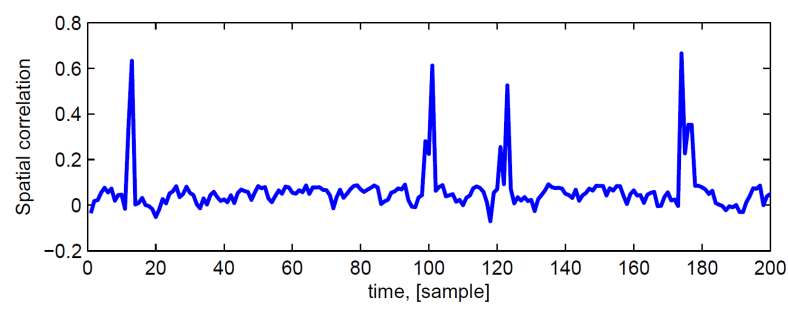

(b) Spatial correlation function $R^{11,22}$

Fig. 2. Simulated statistics over time for double mobile scenario

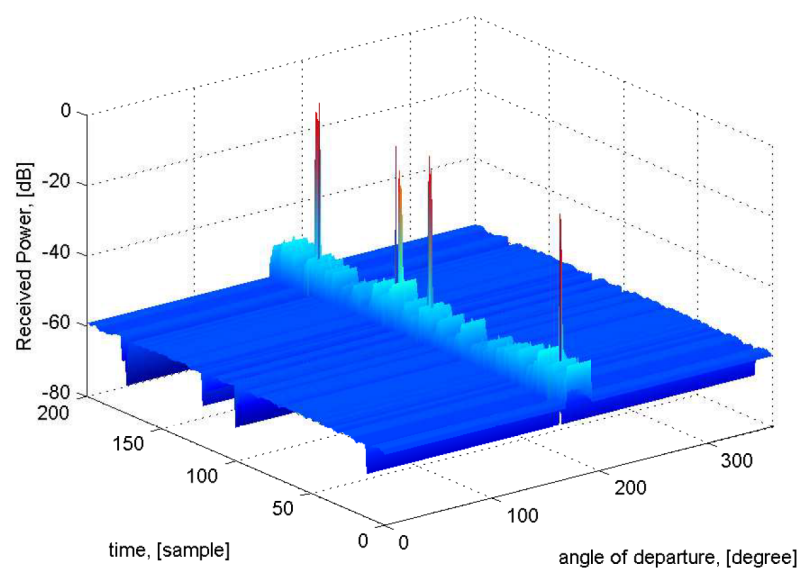

Fig. 3. Simulated Power Angular Spectrum

\section{Model Summary AND Simulation Results}

This section presents some simulation results. In simulations, we use $L_{T}=L_{R}=2$ and $\delta_{T}=\delta_{R}=0.5 \lambda$. An HMM-based method described in [2] is used here to model time-variant fading statistics of an indoor mobile-to-mobile channel. Duration of one state of the HMM (sample) is chosen the same as in [2] and equals $0.8 \mathrm{~s}$. The temporal behavior of the SOSF parameters is shown in Fig. 2 a. Next, using modeled values of the parameters $\alpha$ and $\beta$, the weights in (11) and (15) can be calculated as

$$
\omega_{0}=\sqrt{\beta}=\sqrt{\frac{K}{1+K}}
$$

$$
\begin{gathered}
\omega_{1}=\sqrt{1-\alpha-\beta}=\sqrt{1-\alpha-\frac{K}{1+K}} \\
\omega_{2}=\sqrt{\alpha},
\end{gathered}
$$


where $K$ is the Rician $\mathrm{K}$-factor.

Time-variant weights (extracted from parameters $\alpha$ and $\beta$ modeled by the HMM by using (16) - (18)) are used to calculate theoretical spatial correlation $R^{11,22}(0.5 \lambda, 0.5 \lambda, t)$ (Fig. $2 b$ ) and Power Angular Spectrum (Fig. 3). Next, a pre-defined number of correlated complex fading realizations $h_{p q}(t)$ with appropriate first- and second-order statistics can be generated as a weighted combination of the components (6) - (10) by using any of simulation models found in literature (e.g. [3], [9], [4]).

\section{CONCLUSION}

This paper has presented a model of time-variant fading statistics of MIMO indoor channels: (i) a reference model for second-order scattering fading (SOSF) has been proposed; (ii) probability density functions of angles of departure and arrival have been presented; (iii) the theoretical spatial correlation function for SOSF has been derived and simulated.

\section{ACKNOWLEDGEMENTS}

This research has been funded by the Interuniversity Attraction Poles Programme 7/23 BESTCOM initiated by the Belgian Science Policy Office.

\section{REFERENCES}

[1] C. Oestges, N. Czink, B. Bandemer, P. Castiglione, F. Kaltenberger, and A. Paulraj, "Experimental characterization and modeling of outdoor-toindoor and indoor-to-indoor distributed channels," IEEE Transactions on Vehicular Technology, vol. 59, no. 5, pp. 2253-2265, Jun 2010.

[2] E. Vinogradov, W. Joseph, and C. Oestges, "Measurement-based modeling of time-variant fading statistics in indoor peer-to-peer scenarios," Antennas and Propagation, IEEE Transactions on, vol. 63, no. 5, pp. 2252-2263, May 2015.

[3] A. Zajic and G. Stuber, "Space-time correlated mobile-to-mobile channels: Modelling and simulation," IEEE Transactions on Vehicular Technology, vol. 57, no. 2, pp. 715-726, March 2008.

[4] B. Hogstad, M. Pätzold, N. Youssef, and D. Kim, "A MIMO mobile-tomobile channel model: Part II - The simulation model," IEEE International Symposium on Personal, Indoor and Mobile Radio Communications (PIMRC), pp. 562 - 567, September 2005.

[5] J. Salo, H. El-Sallabi, and P. Vainikainen, "Statistical analysis of the multiple scattering radio channel," IEEE Transactions on Antennas and Propagation, vol. 54, no. 11, pp. 3114-3124, Nov 2006.

[6] B. Bandemer, C. Oestges, N. Czink, and A. Paulraj, "Physically motivated fast-fading model for indoor peer-to-peer channels," Electronics Letters, vol. 45, no. 10, pp. 515-517, May 2009.

[7] Y. Ma and M. Patzold, "A wideband one-ring mimo channel model under non-isotropic scattering conditions," in Vehicular Technology Conference, 2008. VTC Spring 2008. IEEE, May 2008, pp. 424-429.

[8] A. Zajic and G. Stuber, "Space-time correlated mimo mobile-to-mobile channels," in Personal, Indoor and Mobile Radio Communications, 2006 IEEE 17th International Symposium on, Sept 2006, pp. 1-5.

[9] D. Young and N. Beaulieu, "The generation of correlated Rayleigh random variates by inverse discrete fourier transform," IEEE Transactions on Communications, vol. 48, no. 7, pp. 1114-1127, Jul 2000. 